\title{
Electrical triggering of earthquakes: results of laboratory experiments at spring-block models
}

\author{
Victor A. Novikov • Vladimir I. Okunev • Vadim N. Klyuchkin • \\ Jing Liu $\cdot$ Yuri Ya. Ruzhin $\cdot$ Xuhui Shen
}

Received: 17 February 2017/ Accepted: 10 April 2017/Published online: 9 May 2017

(C) The Author(s) 2017. This article is an open access publication

\begin{abstract}
Recently published results of field and laboratory experiments on the seismic/acoustic response to injection of direct current (DC) pulses into the Earth crust or stressed rock samples raised a question on a possibility of electrical earthquake triggering. A physical mechanism of the considered phenomenon is not clear yet in view of the very low current density $\left(10^{-7}-10^{-8} \mathrm{~A} / \mathrm{m}^{2}\right)$ generated by the pulsed power systems at the epicenter depth $(5-10 \mathrm{~km})$ of local earthquakes occurred just after the current injection. The paper describes results of laboratory "earthquake" triggering by DC pulses under conditions of a spring-block model simulated the seismogenic fault. It is experimentally shown that the electric triggering of the laboratory "earthquake" (sharp slip of a movable block of the spring-block system) is possible only within a range of subcritical state of the system, when the shear stress between the movable and fixed blocks obtains $0.98-0.99$ of its critical value. The threshold of electric triggering action is about $20 \mathrm{~A} / \mathrm{m}^{2}$ that is $7-8$ orders of magnitude higher than estimated electric current density for Bishkek test site
\end{abstract}

V. A. Novikov $(\bowtie)$ · V. I. Okunev · V. N. Klyuchkin Joint Institute for High Temperatures of Russian Academy of Sciences, Moscow, Russia

e-mail: novikov@ihed.ras.ru

J. Liu

Institute of Earthquake Science, China Earthquake

Administration, Beijing, China

Y. Ya. Ruzhin

Pushkov Institute of Terrestrial Magnetism and Radio Wave Propagation, Troitsk, Moscow Region, Russia

X. Shen

Institute of Crustal Dynamics, China Earthquake Administration, Beijing, China
(Northern Tien Shan, Kirghizia) where the seismic response to the man-made electric action was observed. In this connection, the electric triggering phenomena may be explained by contraction of electric current in the narrow conductive areas of the faults and the corresponding increase in current density or by involving the secondary triggering mechanisms like electromagnetic stimulation of conductive fluid migration into the fault area resulted in decrease in the fault strength properties.

Keywords Earthquake simulation · Spring-block model · Electric pulse $\cdot$ Triggering threshold

\section{Introduction}

In the middle of 1990s, a statistically significant impact of electrical pulses of direct current (DC) current injected into the Earth crust through the grounded dipole on the spatial and temporal distribution of local weak seismicity of Pamir (Tarasov 1997) and Northern Tien Shan (Tarasov et al. 2000) regions has been discovered. The grounded electrical dipole of about $4 \mathrm{~km}$ length was supplied by single electrical pulses of DC current of 1.5-2.5 kA, voltage of $1 \mathrm{kV}$ and duration of 2.5-10.0 s from geophysical magneto-hydrodynamic (MHD) generator used for deep electromagnetic (EM) sounding of the earth crust for monitoring of the crust stress state and search of EM earthquake precursors. It was statistically found that after injection of electric current into the earth crust the number of weak earthquakes $\left(m_{\mathrm{b}}<3.0\right)$ was increased by several times with a time delay of 1-2 days, and then, within 5-7 days, was returned to the previous background level. A hypothesis on a triggering action of electrical pulses has been proposed 
(Zeigarnik et al. 1999). Laboratory experiments carried out to date with application of various press equipment and samples of geomaterials under stress-strain state demonstrated a modulation of acoustic emission by electric current pulses applied to the sample (Sobolev and Ponomarev 2003; Bogomolov et al. 2004; Avagimov et al. 2006). It was shown that a response of acoustic emission to electric action characterized crack formations in the sample rises as the sample loading and maximally appears at the stress level of $0.95-0.99$ of breaking stress of the material (Sobolev and Ponomarev 2003). The effect of modulation of geoacoustic emission by external actions is well known, e.g., under impact of lunar-solar earth tides (Gordeev et al. 1996). Nevertheless, a response of acoustic emission to the external action does not mean that it can unambiguously result in the macro-event (earthquake or failure of tested rock sample) triggering (Beeler and Lockner 2003). A purpose of the presented study is an experimental determination of conditions for laboratory "earthquake" triggering by a pulse of electric current, as well as a triggering potential/threshold of the electrical impact.

\section{Experimental method}

In contrast to previous experiments with specialized press equipment (Sobolev and Ponomarev 2003; Bogomolov et al. 2004; Avagimov et al. 2006), when a response of acoustic emission to electrical action on the tested sample was studied, the presented research employs a spring-block facility (Fig. 1) based on a model of earthquake as an unstable sliding with friction on a contact of rock blocks (Brace and Byerlee 1966). This facility allows to study not only behavior of acoustic emission (formation of cracks) under electrical actions, but also a possibility of triggering the macro-event-sharp sliding of the movable block (item 1 in Fig. 1) called as laboratory "earthquake". The springblock tribological facility (Fig. 1), where an interface between the movable block (1) and the fixed block (2) represents the contact of fault edges, simulates a seismic cycle: accumulation and release of shear stresses in the fault. The contact area between the fixed and movable blocks where the shear stresses are accumulated is filled by granulated material: the fault gouge (3), which simulates dispersed rocks occurred during the crashing the fault edges. The vertical load of the movable block (1) provides a normal pressure in the contact area between the blocks. The electromechanical drive (5) provides slow loading of the spring (4) connected with the movable block (1) with $0.13 \mu \mathrm{m} / \mathrm{s}$ linear velocity. When the shear stress in the contact area reaches its critical value, the contact fails and sharp slip of the movable block occurs. The detailed description of the spring-block facility and diagnostic equipment can be found in Kocharyan and Novikov (2016), Kocharyan et al. (2017). This facility employs the system of electrical action on the fault area, which provides a simulation of electric current through the fault for a study of its possible triggering impact on the seismic process.

For the presented tests, a movable block of $227 \times 112 \mathrm{~mm} \times 53 \mathrm{~mm}$ and a fixed block of 800 , $500 \mathrm{~mm} \times 80 \mathrm{~mm}$ were manufactured from concrete of $1974 \mathrm{~kg} / \mathrm{m}^{3}$ density. The copper plate electrodes (Fig. 1, item 11) for electric current injection into the simulated fault gouge were imbedded flush on the bottom surface of the movable block. For simulation of electric resistivity of the fault gouge under natural conditions, the gouge granular material and the blocks were water-saturated. For the simulated fault gouge, the wet quartz sand with grain sizes of $0.2-0.5 \mathrm{~mm}$ and electric conductivity of $3.75 \mathrm{~S}$ was used. The gouge thickness after compaction of quartz sand

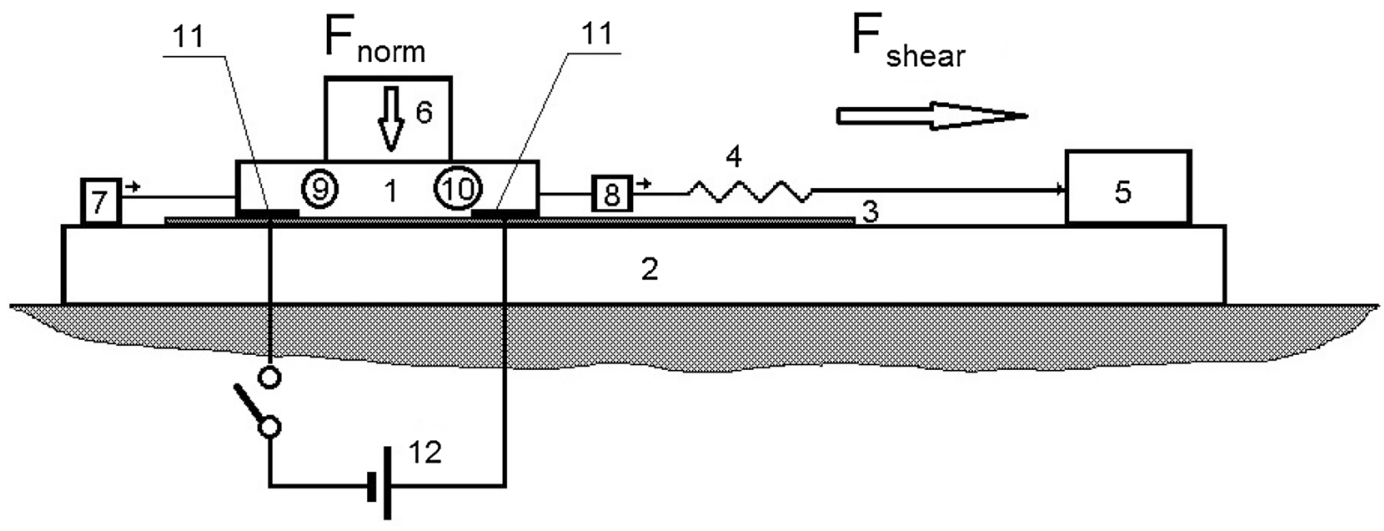

Fig. 1 Functional diagram of the spring-block facility with a system of electric pulses injection into contact area between the movable and fixed blocks. 1 Movable block, 2 fixed block, 3 contact area gouge (granulated material), 4 spring, 5 electromechanical drive, 6 normal load of the contact area, 7 motion transducer of the movable block, 8 shear force transducer of the movable block, 9 sensor of high-frequency acoustic emission, 10 sensor of low-frequency acoustic emission, 11 electrodes embedded into the bottom part of the movable block, 12 DC current source 
during the preliminary test was $\sim 1.1 \mathrm{~mm}$. Electric conductivity of the wet concrete blocks was much less $(1.2 \times$ $10^{-3} \mathrm{~S}$ ) and can be neglected when calculating the current density in the granulated gouge layer. The normal load of the contact area was $113.76 \mathrm{kgf}$ that provided the normal stress of $0.45 \mathrm{kgf} / \mathrm{cm}^{2}$ in the granulated gouge. The stiffness of calibrated spring was $1.7 \mathrm{kgf} / \mathrm{mm}$. At the first stage of the tests, the conditions were determined for provision of regular seismic cycles: "stick-slip" mode (slow accumulation and sharp release of shear stress in the contact area). When the shear force reached a critical value $F_{\max }$, a sharp slip of the movable block occurs. A series of ten regular slips was performed, and the average critical shear force was estimated as $F_{\max }=31.5_{-0.31}^{+0.13} \mathrm{kgf}$. At the second stage of the tests, the spring-block system by slow spring loading was driven into subcritical state where the shear force reached a value of $0.95-0.98 F_{\max }$. After that, the electromechanical drive of the spring-block system was switched off for the period of a few seconds when the system state was monitored by the measured intensity of acoustic emission. Then, when the acoustic emission obtained the steady-state value, an electric action to the contact area was provided by DC electrical pulses from the pulse generator AHP-1120 with rectangular pulse frequency of $200 \mathrm{~Hz}$. The DC pulse amplitude was varied in a range of 1-7 mA. The following parameters of the test system were recorded: spring force (UMMA-K100 transducer), block movement (sensor CTE-3000), high-frequency acoustic emission (AE) (sensor PAE PG20-200; frequency range of 20-200 kHz), low-frequency AE (sensor AP57, frequency range of $1 \mathrm{~Hz}-$ $2 \mathrm{kHz}$ ), electric current (DC current recorder embedded into the power amplifier). The analog signals from the sensors and transducers were transmitted to analog-digital converter USB-3000 with the following processing of digital data by PowerGraph ${ }^{\odot}$ software package.

\section{Experimental results}

After preliminary drawing of the movable block and compaction of the gouge between the blocks, two series of tests were conducted: the first series of tests without electric current (observation of ten regular slips for determination of critical shear force $F_{\max }$ ) and the second series of tests with electric current injection into the simulated fault (80 tests) for different subcritical state of the system (shear force $F_{\text {test }}=0.95-0.99 F_{\max }$ ) and different electric current of 1-7 mA. The test conditions are summarized in Table 1 .

Parameters of the typical slip in the test without electric action to the contact area are shown in Fig. 2. The spring force $F$ continuously rises up to the block slip occurrence at
$F_{\max }=31.85 \mathrm{kgf}$. After the block slip, the spring force drops to $F_{\min }=28.87 \mathrm{kgf}$, and the block displacement is $\Delta S=3.5 \mathrm{~mm}$. Just $2.86 \mathrm{~s}$ before the slip AE amplitude and intensity started increasing (see AE HF sensor reading, Fig. 2), the movement sensor recorded the block microslip, and the spring-block system passed into an unstable state. AE rose to the maximal values at $F_{\text {max }}$, and after the failure and stop of the movable block, it returned to the background values.

An example of triggering the block slip by electric impact is shown in Fig. 3. The tests with electric action were performed in four stages. At the first stage, the spring force raised up to $0.987 F_{\max }$, after that the electromechanical drive was switched off, and the second stage (pause in the block loading) was started with duration of $15.4 \mathrm{~s}$. At the third test stage, the electric action on the contact area between movable and fixed blocks was performed (drive was still switched off). During the electric action stage, DC current was injected into the block interface via electrodes and the wet fault gouge during $15 \mathrm{~s}$ (Fig. 2). Depending on the amplitude of DC pulses, the slip was not triggered or triggered with some time delay. It should be noted that after beginning of the electric action a rise of $\mathrm{AE}$ and a drop of $F$ from 31.52 to $31.46 \mathrm{kgf}$ were observed before occurrence of the slip triggering. In so doing, when the electric current is supplied into the block interface, some small relaxation of shear stress occurs, that transfers an avalanche-like $\mathrm{AE}$ rise into sharp slip of the block (laboratory "earthquake"). The DC pulse amplitude was varied from 1 to $7 \mathrm{~mA}$ (Table 1), and the slip triggering was observed at DC value over $5 \mathrm{~mA}$, which may be considered as the threshold value of electric action. It should be noted that the slip triggering does not occur when the electric action is performed at the shear force less than 0.98. In this case, AE gradually decreases and obtains the background level without the block slip. An increase in duration of electrical action even to $55 \mathrm{~s}$ does not initiate the slip. The slip occurrence and its duration are considered as the fourth stage of the test. The slip duration was similar to slip without electrical action (0.39-0.55 s). Considering the gouge cross-sectional area $\left(227 \times 2 \mathrm{~mm}^{2}\right)$ between electrodes and current losses $\Delta I$ due to current spreading through the gouge before and after the movable block ( $\Delta I=1.65 \%$ : experimental value), the threshold current density for laboratory "earthquake" triggering can be estimated. Keeping in mind electrical conductivity of the wet gouge material $(3.75 \mathrm{~S})$ and concrete used for the blocks $\left(1.2 \times 10^{-3} \mathrm{~S}\right)$, the current losses in the concrete blocks may be neglected. In our case, the threshold current density is $5 \mathrm{~mA}$ (minimal current for slip triggering) $\times 0.9835$ (current losses $) /\left(227 \times 1.1 \mathrm{~mm}^{2}\right)=19.7 \mathrm{~A} / \mathrm{m}^{2}$. 
Table 1 Number of slips for tests without and with electric current injection into the simulated fault

\begin{tabular}{llllll}
\hline Electric current $(\mathrm{mA})$ & $F_{\max }(\mathrm{kgf})$ & \multicolumn{5}{l}{ Shear force $F_{\text {test }}(\mathrm{kgf})$} & for electric current injection \\
\cline { 3 - 6 } & $31.5_{-0.31}^{+0.13}$ & $29.93\left(0.95 F_{\max }\right)$ & $30.56\left(0.97 F_{\max }\right)$ & $30.87\left(0.98 F_{\max }\right)$ & $31.19\left(0.99 F_{\max }\right)$ \\
\hline 0.0 & 10 & - & - & - & - \\
1.0 & - & $5^{*}$ & $5^{*}$ & $5^{*}$ & $5^{*}$ \\
3.0 & - & $5^{*}$ & $5^{*}$ & $5^{*}$ & $5^{* *}$ \\
5.0 & - & $5^{*}$ & $4^{* / 11^{* *}}$ & $2^{* / 3 *}$ & $5^{* *}$ \\
7.0 & - & $5^{*}$ & $3^{* *} 2^{* *}$ & \\
\hline
\end{tabular}

* Number of tests without slip triggering by electric current

** Number of tests with slip triggered by electric current

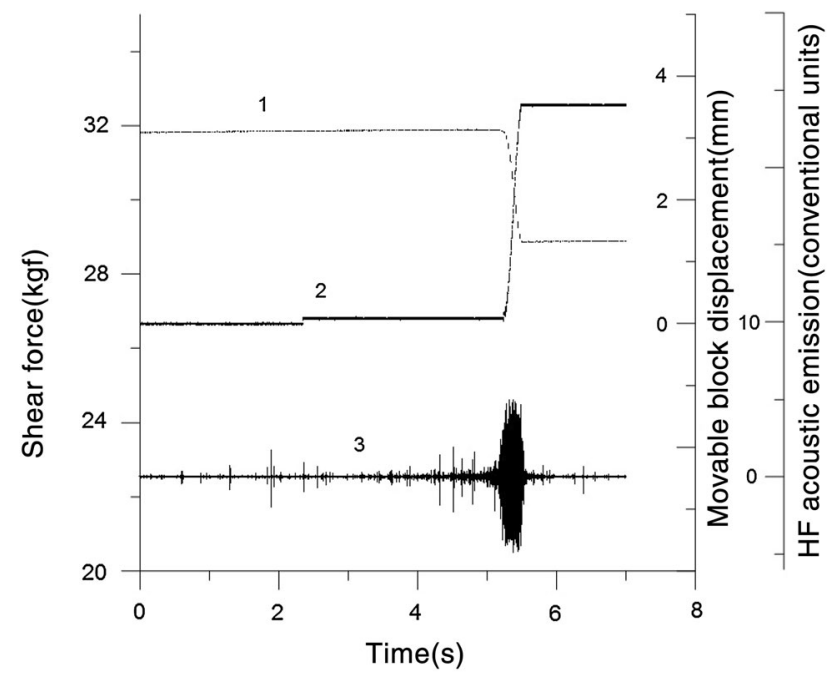

Fig. 2 Variation of parameters of the spring-block facility during preparation and occurrence of sharp slip of the movable block over the fixed block (laboratory "earthquake") without electric action on the contact area. 1 shear force of the movable block, 2 movable block displacement, 3 high-frequency acoustic emission

\section{Discussion}

The above-described laboratory experiments demonstrate a possibility of direct triggering of seismic event by pulses of DC current, which is injected along the fault area. Here, the triggering of fault failure by electrical action is possible only in the subcritical shear stress range, when the accumulated shear stresses are $0.98-0.99$ of critical value corresponded to occurrence of sharp slip (laboratory "earthquake"). It should be noted that the threshold DC current density, which provides the seismic event triggering, is about $20 \mathrm{~A} / \mathrm{m}^{2}$ that exceeds approximately by $7-8$ orders the current density at the depth of earthquake source $(5-10 \mathrm{~km})$ calculated for Bishkek test site, when a response of regional seismicity on the pulses of DC current injected into the Earth crust through the grounded electric dipole supplied by pulsed MHD generator is analyzed. In spite of

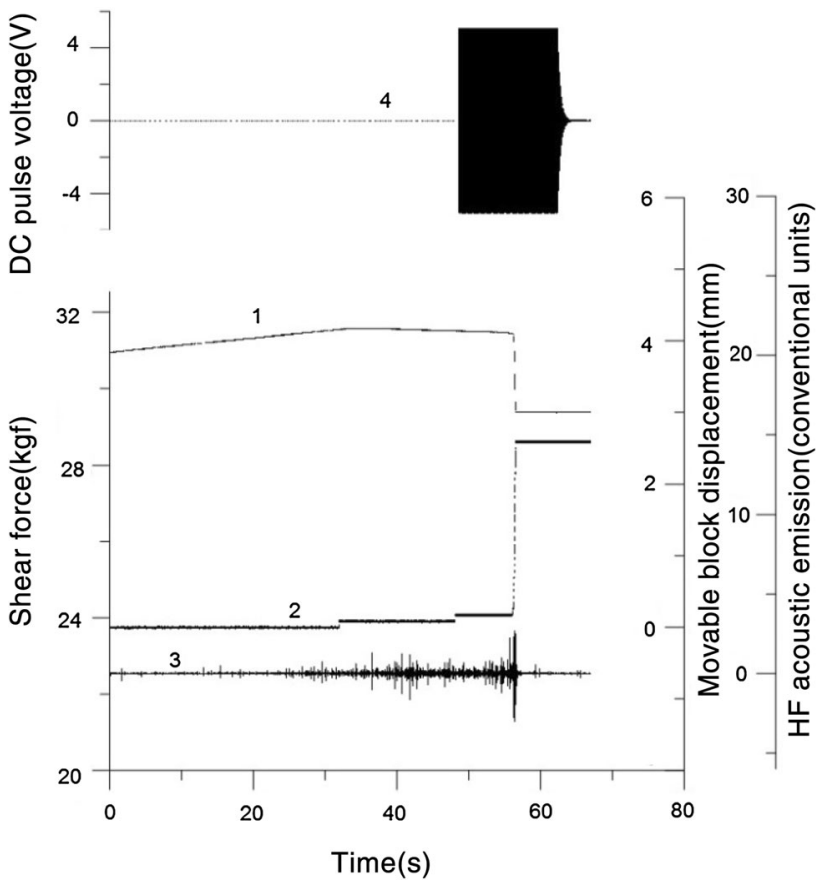

Fig. 3 Variation of parameters of the spring-block facility during preparation and triggering of sharp slip of the movable block by electrical action (electrically triggered laboratory "earthquake"). 1 shear force of the movable block, 2 movable block displacement, 3 high-frequency acoustic emission, 4 voltage of electric action (EA)

clear evidence of spatiotemporal redistribution of the local seismicity after electrical processing of the Earth crust by different electric power sources provided DC current in emitting dipole of 600-2500 kA, the mechanism of such response of stressed rocks to weak electrical impact is unknown. For both field and laboratory experiments, when the electric action duration is of $10 \mathrm{~s}$ order, the Joule heating of the medium, even for current density of 20 $\mathrm{A} / \mathrm{m}^{2}$, is negligible low, and cannot be considered as a reason of decrease in efficient strength of rocks in the fault due to the increase in fluid pore pressure provided by electrical heating. 
When the other mechanisms are considered, e.g., generation of double electric layers in the medium as a result of external electric action, which may develop additional stresses in the rock massif (Zeigarnik et al. 1999), the following should be taken into account. An analysis of field observations of dynamic earthquake trigging (Gomberg et al. 1998) shows that the triggering threshold of dynamical triggering from the distant strong earthquakes is on the level of $\sim 0.1 \%$ of lithostatic pressure in the earthquake source. Therefore, an additional triggering force created by some external physical action should perform some mechanical work $A_{\text {mech }}$ for compression of $1 \mathrm{~m}^{3}$ of rock, which may be estimated as follows:

$A_{\text {mech }}=\Delta F \cdot \Delta \delta \cdot 1 \mathrm{~m}=\Delta F \cdot L \cdot \Delta P / E=2 \times 10^{5} \mathrm{~N} \quad \times$ $1 \mathrm{~m} \times 0.2 \mathrm{MPa} / 5 \times 10^{4} \mathrm{MPa}=0.8 \mathrm{~J}$, where $\Delta F$ is an additional force due to variation of lithostatic pressure by $0.1 \%$, acting on a surface of the block of $1 \mathrm{~m}^{2}$ area is $0.2 \mathrm{MPa} \times 1 \mathrm{~m}^{2}=2 \times 10^{5} \mathrm{~N} ; L$ is a length of the compressed block, and $L=1 \mathrm{~m} ; \Delta \delta=\Delta P / E$ : relative deformation of the block at $\Delta P=0.2 \mathrm{MPa}$ (variation of normal stress $\sim 200 \mathrm{MPa}$ at the depth of $10 \mathrm{~km}$ by $0.1 \%$ ), $E=5 \times 10^{4} \mathrm{MPa}$ is an elastic modulus of granite.

The work provided by electric current during $10 \mathrm{~s}$ (maximal duration of DC current pulse in field experiments) through the conductor with specific resistance of $5 \times 10^{3} \mathrm{Ohm} \cdot \mathrm{m}$ (averaged value for Bishkek geodynamical test site) of $1 \mathrm{~m}^{2}$ area and $1 \mathrm{~m}$ length will be $A_{\mathrm{el}}=I^{2} \cdot R \cdot t=\left(1 \times 10^{-8}\right) \mathrm{A}^{2} \times 5 \times 10^{3} \Omega \cdot \mathrm{m} \times 1 \mathrm{~m} / 1 \mathrm{~m}^{2}$ $\times 10 \mathrm{~s}=5 \times 10^{-14} \mathrm{~A}^{2} \mathrm{Ohm} \cdot \mathrm{s}=5 \times 10^{-14} \mathrm{~J}$, that is greatly less than the specific work required for earthquake triggering by mechanical action $\left(\sim 1 \mathrm{~J} / \mathrm{m}^{3}\right)$.

In so doing, even the whole work performed by electric current will be converted into mechanical energy of rock compression, it will be not enough for provision of required variation of normal stress in the fault by $0.1 \%$, which can trigger earthquake. Based on provided above estimations, the conclusion may be drawn that for field experiments with pulsed MHD generators even for $100 \%$ conversion of electrical energy into mechanical work in the earthquake source (though the most part of electrical energy will be converted into heat) the generation of required additional stresses of $0.05-0.2 \mathrm{MPa}$ capable to trigger earthquake is impossible and another hypotheses should be considered for explanation of electric triggering phenomena observed under field conditions. For obtaining the required level of increase in specific energy of rock $\left(1 \mathrm{~J} / \mathrm{m}^{3}\right)$, the electric current density should be about $0.2 \times 10^{-2} \mathrm{~A} / \mathrm{m}^{2}$ and, considering the heat losses, even greater, that in principle meets the obtained laboratory test results. Thus, the effect of seismicity response to electrical pulses observed under natural conditions may be explained either by contraction of electric current in narrow highly conductive fault areas and current density increase or by secondary triggering mechanisms like electromagnetic stimulation of fluid migration into the fault area resulted in decrease in the fault strength properties (Novikov and Novikova 2014), that requires special analysis of fluid behavior in the fault and related variations of electrical and mechanical properties of the fault gouge.

\section{Conclusions}

Thus, in the laboratory experiments a possibility of direct triggering of seismic event by a pulse of DC current along the fault zone was confirmed. In this case, the triggering of slip of movable block of the spring-block model of seismogenic fault is possible only in the subcritical state of the spring-block system, when the accumulated shear stresses are 0.98-0.99 of critical value, when the sharp slip (laboratory "earthquake") occurs. The threshold current density triggered the seismic event under laboratory conditions is about $20 \mathrm{~A} / \mathrm{m}^{2}$, that exceeds approximately by 8 orders the current density calculated for field experiments carried out at the Bishkek geodynamical test site (Northern Tien Shan, Kirghizia), when the seismic response to electrical pulses injected into the Earth crust by MHD generator is analyzed. The effect of local seismicity response to electrical action may be explained either by electric current contraction in the narrow highly conductive fault zones up to the current density required for earthquake triggering (over $0.2 \times 10^{-2} \mathrm{~A} / \mathrm{m}^{2}$ ) or by secondary triggering mechanisms driven by electric pulses, like electromagnetic stimulation of fluid migration into the fault zone and reduction of the fault strength properties.

Acknowledgements The reported study was funded by Russian Foundation for Basic Research according to research project No. 15-55-53104 and by National Natural Science Foundation of China according to International cooperation project No. 41511130032 . We thank for constructive comments of anonymous referees, which improved the manuscript.

Open Access This article is distributed under the terms of the Creative Commons Attribution 4.0 International License (http://crea tivecommons.org/licenses/by/4.0/), which permits unrestricted use, distribution, and reproduction in any medium, provided you give appropriate credit to the original author(s) and the source, provide a link to the Creative Commons license, and indicate if changes were made.

\section{References}

Avagimov AA, Zeigarnik VA, Klyuchkin VN (2006) On the structure of acoustic emission of model samples in response to an external energy action. Phys Solid Earth 42(10):824-829

Beeler NM, Lockner D (2003) Why earthquakes correlate weakly with the solid Earth tides: effects of periodic stress on the rate and probability of earthquake occurrence. J Geophys Res Solid Earth 108(B8):2391 
Bogomolov LM, Il'ichev PV, Novikov VA, Okunev VI, Sychev VN, Zakupin AS (2004) Acoustic emissions response of rocks to electric power action as seismic-electric effect manifestation. Ann Geophys 47(1):65-72

Brace WF, Byerlee JD (1966) Stick-slip as a mechanism for earthquakes. Sci New Series 153(3739):990-992

Gomberg J, Beeler NM, Blanpied ML (1998) Earthquake triggering by static and dynamic deformations. J Geophys Res 103:24411-24426

Gordeev EI, Saltykov VA, Synitsyn VI, Chebrov VN (1996) Correlation of high-frequency noise with lunar-solar tides. Dokl Earth Sci 343A(6):42-45

Kocharyan GG, Novikov VA (2016) Experimental study of different modes of block sliding along interface. Part 1. laboratory experiments. Phys Mesomech 19(2):189-199

Kocharyan GG, Novikov VA, Ostapchuk AA, Pavlov DV (2017) A study of different fault slip modes governed by the gouge material composition in laboratory experiments. Geophys $\mathbf{J}$ Int 208(1):521-528

Novikov V, Novikova E (2014) Electromagnetic stimulation of fluid migration into fault area and earthquake triggering phenomena. Geophys Res Abstr 16: EGU201-14-12790

Sobolev GA, Ponomarev AV (2003) Earthquake physics and precursors. Nauka, Moscow (in Russian)

Tarasov NT (1997) Crustal seismicity variation under electric action. Dokl Acad Nauk SSSR 353(4):542-545

Tarasov NT, Tarasova NV, Zeigarnik VA, Avagimov AA (2000) The effect of high energy electromagnetic pulses on seismicity in Central Asia and Kazakhstan. J Volcanol Seismol 21(4-5): $627-639$

Zeigarnik VA, Tarasov NT, Avagimov AA (1999) Managing earthquakes? Science in Russia 2:16-21 